\title{
Pathogenesis of Influenza
}

\section{Jian Wang}

Influenza virus can infect various types of cells in human respiratory tract and replicate inside. Viral replication damages its infected cells to cause their apoptosis, which is the underlying mechanism of its pathogenesis. Once influenza virus gains its access to human respiratory tract, it adheres to and penetrates into the epithelial cells for its replication there for 4-6 h. The newly emerging virus particles bud from cell membrane and are released with assistance by neuraminidase to infect adjacent epithelial cells. In such a way, a large quantity of epithelial cells are infected within a short period of time. The infected epithelial cells are then subjected to apoptosis and shedding to cause local inflammatory response as well as systemic toxic symptoms such as acute onset of high fever, systemic soreness and pain, and decreased WBC count. The infected cells can also produce excessive interferon, which may be related to the systemic symptoms. However, viremia does not occur.
The pathological changes of simplex influenza include impaired upper and middle respiratory tract, obviously involved trachea, degenerated ciliated epithelial cells with apoptosis and shedding, detectable inclusion bodies in the cytoplasm, mucosal congestion and edema as well as infiltrated mononuclear cells. However, the layer of basal cells remains intact. About 4-5 days after onset, the basal cells begin to proliferate to form undifferentiated epithelial cells. Two weeks later, ciliated epithelial cells are formed for recovery. Pneumonia induced by influenza virus is pathologically characterized by intrapulmonary extensive hemorrhage in a color of dark red with accompanying edema, bloody secretions in the trachea and bronchi, mucosal congestion, necrosis and shedding of tracheal and bronchial ciliated epithelial cells, submucosal focal hemorrhage, edema and slight inflammatory cells infiltration as well as alveolar fibrin exudates containing neutrophils and mononuclear cells.

J. Wang

Affiliated First Hospital, Xinjiang Medical University,

Urumuqi, Xinjiang, China

e-mail: jeanw1265@163.com 